\title{
A Study of Blood Lactic Acid and Isokinetic Muscular Strength after Maximal Load Exercise
}

\author{
Dongyup Han ${ }^{1}$, BYunguun $\mathrm{CHO}^{2)}$ \\ 1) Department of Physical Education, Chungnam National University \\ 2) Department of Emergency Medical Technology, Kangwon National University: Kuydong Samchuock \\ city, Kangwondo, 245-711, South Korea.TEL: +82 33-570-6440,E-mail: cho6451@gmail.com
}

\begin{abstract}
Purpose] This study was undertaken to understand the effects of three different ways of resting after maximal exercise on lactic acid in blood, and muscular strength. [Methods] To achieve this objective, nine athletes were selected as blood donors. Blood was gathered from each donor at three different times: at rest, after maximal exercise, and after recovery. Strength was measured after 30 minutes maximal exercise. [Results] Lactic acid of significant differences appeared more in the walking + jogging rest than the national gymnastics rest and was more effective in removing lactate. [Conclusion] Isokinetic strength (Peak Torque, Peak Torque \% Body Weight) were significantly better after the walking + jogging rest than after the national gymnastics rest.

Key words: Maximal exercise, Lactic acid, Muscular strength
\end{abstract}

(This article was submitted Nov. 29, 2011, and was accepted Jan. 19, 2012)

\section{INTRODUCTION}

Most team sports are divided into halves, quarters or time-outs, during which players are provided with an intermittent break. In this span of time, they expect strategic instructions and physical recovery. The objective of taking a break is to restore the human body to a stable state. A sudden stop of exercise may occasionally cause the feeling of nausea, dizziness and acute fatigue, because after a sudden stop in exercise, blood circulation rapidly drops, and blood flow from the heart decreases 1,2 .

Studies of changes in blood lactate and lactate dehydrogenase, depending on maximum exercise intensity have been implemented, and such studies are frequently conducted in order to review exercise prescription and training effects on athletes. Following a high-load exercise, creatinekinase, CK, a blood fatigue substance, was found to remarkably increase. CK catalyses the Lohmann reaction which combines phosphocreatine, with adenosine diphosphate, and then recompounds it with adenosine triphosphate. In addition, $\mathrm{CK}$ is reported to act as a blood marker of polymyositis, skin reaction, skeletal muscle disease, and it increases after high-load muscle resistance exercise, in particular, following eccentric contraction 月. $^{2}$.

This study investigated the dissipation and reduction of muscle pain or muscle spasms of normal persons after workout and muscle fatigue of elite athletes following exercise. The removal of lactate from the muscle and blood can be of potential merit for elite athletes. In general, typically suggested cool-downs are a low intensity workouts of the main training, such as walking, jogging, stretching exercise, and recreational games, for example $\$$.

Exercise recovery which is accompanied by light workouts including jogging requires a higher intake of air than rest recovery, so recovery is faster. Regarding the speed of cool-down, $65 \%$ of maximum exercise speed is efficient, which is the speed that swimmers feel most comfortable with $\$$. It is reported that general cool-downs, which athletes mostly perform, but they do focus on exercises that help physical function and cardiopulmonary function, not help muscle pain or muscle recovery . However, the study of which resting method improves athletic performance during a short break in a game is lacking.

The purpose of this study was to formulate a resting program which prevents the increase of the rate of lactate recovery following maximum load exercise by elite athletes and prevents the decrease of muscle performance due to after-exercise fatigue, as well as to provide basic data for enhancing of athletic performance and the prevention injury.

\section{SUBJECTS AND METHODS}

The subjects were 9 men with over 5 years of experience as ball game players who had no injury of the knees or ankles or other physical injuries. The physical characteristics of the subjects were as follows: age, $20.36 \pm 1.28$ years; height, $182.32 \pm 3.68 \mathrm{~cm}$; weight, $74.21 \pm 1.95 \mathrm{~kg}$.

This study used three types of resting methods following maximum exercise. A Type was static rest, B Type was national gymnastics, which is a temporarily conducted cool-down. C Type was a walking + jogging rest program which was designed by the authors. C Type employs relatively large and unequal increments i.e., METs/stage every 3 minutes (Bruce protocol). To measure the change of muscle strength, we measured Peak Torque, and Peak Torque \%Body Weight, which are the measuring factors of 
Table 1. The recovery rates of blood lactate concentration

\begin{tabular}{ccc}
\hline $\mathrm{A}$ & $\mathrm{B}$ & $\mathrm{C}$ \\
\hline $52.01 \pm 7.24$ & $68.58 \pm 6.42$ & $78.72 \pm 8.21^{*}$ \\
\hline (Unit:\%) ${ }^{*}$ p $<0.05$ & $\mathrm{~A}:$ static rest, B: national gymnastics, C: \\
walking + jogging
\end{tabular}

isokinetic muscle strength of flexor muscles and extensor muscles. The exercise speed was individualized so that exhaustion occurred in approximately 8-12 minutes 8 . Maximum exercise load was reached at an all-out state using the Bruce protocol on a treadmill, and blood samples for measuring blood lactate concentration were collected by the fingertip method at $3 \mathrm{~min}, 5 \mathrm{~min}, 15 \mathrm{~min}$ and $30 \mathrm{~min}$ after exercise, and immediately analyzed using a YSI 1500. Cyber-770 (Lumax. U.S.A.) was used to measure the muscle strength after the cool-down program had been performed, and the maximum muscular strength was measured 5 times at the load of $60 \% \mathrm{sec}$. Subjects conducted a warm-up of 3-5 min before measurement, and warm-ups were implemented three times at a designated load in order to minimize repulsion or unfamiliarity with the machine. Prior to the experiment, the subjects were asked to sit on the seat of a CYBEX exercise machine, and the central axis of the knee joint was positioned on the pivot of the dynamometer. The femoral region, the waist and the chest were fastened by belts in order for the quadriceps femoris muscle and the hamstring muscle not to be affected by external forces during extension of the thigh and the curvature movement. The ankles were fixed by Velcro straps which were adjusted according to the length of the calf using the adjusting arm in order to display the leg strength, which was measured last. The angle between the back of the chair and the coxa, was fixed at $90^{\circ}$, centered on the coxa, and both hands were fastened tp the arm rests of the chair, so that the hands could exert maximum power. To prevent variations in measurements of the calf during flexion and extention, the correct gravity of the machine was adjusted following Lumax regarding the weight of the calf. During measurements, subjects were encouraged by a researcher standing alongside to exert maximal volitional power, and every measurement of the subjects was taken by one researcher to ensure consistency of measurement.

One-way ANOVA was used in order to test the differences in the lactate recovery rates and muscle strengths pursuant to the cool-down programs. SPSS was used for the statistical analyses, and significance was accepted at values of $\mathrm{p}<0.05$.

\section{RESULTS}

The 3 types of resting methods were used by the subjects on different weeks to determine the best recovery rates of lactate and muscle strength (Table 1). The recovery rate of lactic acid in blood was significantly better in the walking + jogging rest type than in the static or national gymnastics rest types, $78.72 \pm 8.21 \%(\mathrm{p}<0.001)$.

Peak Torque and Peak Torque \%Body Weight of the flexor muscles showed no meaningful differences among the rest types at the angular velocity load of $60 \% \mathrm{sec}$, but they displayed a tendency to increase in all rest types (Table 2).

No meaningful differences among the rest types were found in the Peak Torque of the extensor muscles at the angular velocity load of $60 \% \mathrm{sec}$. Peak Torque \%Body Weight of extensor muscles showed no meaningful differences among the rest types at the angular velocity load of $60 \%$ sec.

\section{DISCUSSION}

This study investigated how rest methods influence the recovery of lactate and muscle strength after athletes performed maximum load exercises. It is known that the accumulation of blood lactate concentration during exercise has a strong relationship with the intensity of exercise load, and has a deep influence on muscle fatigue, making it a keen topic of research. Many researchers use the accumulation of blood lactate concentration as an index of exercise load intensity in various sports $\$$. In a previous study, Lee WJ reviewed the influence of the type of after-exercise rest on lactate concentration, and suggested that a more meaningful difference was found in the rate of lactate recovery as time passed after exercise in static rest than in dynamic rest 19.

This suggests that, the walking + jogging rest type would be as more effective removing lactate than the static type. The lactate recovery was significantly better in walking + jogging rest than in static recovery $(\mathrm{P}<0.05)$ at the rest stage of $10 \mathrm{~min}$ and $15 \mathrm{~min}$ following the gradual maximum load exercise. In the case of maximum exercise, it is known that blood lactate which accumulates in the first stage of exercise, drops to the level of the rest time when constant was investigated exercise is performed. The removal of blood lactate during maximum load exercise using a radioactive isotope and the removal speed of lactate was faster when exercise was performed during rest than when the static rest was taken 1 . Furthermore, Lee reported that lactate was removed more rapidly through rest with light exercise recovery than rest recovery 19 . Cool down of sports players has focused on

Table 2. The changes in isokinetic muscle strength

\begin{tabular}{lccc}
\hline Group & A & B & C \\
\hline Peak Torque of flexor (Nm) & $133.78 \pm 18.73$ & $131.22 \pm 17.92$ & $141.77 \pm 18.25$ \\
Peak Torque \%Body Weight of flexor(\%) & $173.77 \pm 22.09$ & $173.10 \pm 20.10$ & $185.40 \pm 21.88$ \\
Peak Torque of extensor (Nm) & $210.89 \pm 30.66$ & $232.56 \pm 17.07$ & $234.56 \pm 21.23$ \\
Peak Torque \%Body Weight of extensor(\%) & $274.72 \pm 32.60$ & $300.64 \pm 32.30$ & $305.64 \pm 38.22$ \\
\hline
\end{tabular}

*:p<0.05 A: static rest, B: national gymnastics, C: walking + jogging 
smooth performance do physical function and the cardiopulmonary function, which do not assist muscle pain dynamic muscular power recovery 1 \%.

In the present study, Peak Torque and Peak Torque \%Body Weight of flexor muscles showed no meaningful differences at the angular velocity load of $60^{\circ} / \mathrm{sec}$, but they displayed a tendency of increase in rest with national gymnastics and rest with walking + jogging.

No meaningful differences among the rest types were found in the Peak Torque in the extensor muscles at the angular velocity load of $60 \% \mathrm{sec}$. Peak Torque \%Body Weight of extensor muscles showed no meaningful differences among the rest types at the angular velocity load of $60 \%$ sec. These results are in agreement with a previous research result ${ }^{11}$.

In conclusion, isokinetic strength of Peak Torque and Peak Torque \% Body Weight showed greater significant differences from static rest in rest with walking + jogging than in rest with national gymnastics. These results indicate the most effective rest method athletes will be able to use in order to reduce muscle fatigue and damage.

\section{REFERENCES}

1) Stamford BA, Weltman A, Moffatt R, et al.: Exercise recovery above and below anaerobic threshold following maximal work. J Appl Physiol, 1981, 51: 840-844. [Medline]

2) ACSM: ACSM's Guidelines for Exercise Testing and Prescription by American College of Sports Medicine $8^{\text {th }}$ edition. Philadelphia: Lippincott Williams \& Wilkins, 2009, pp 1-156.

3) Davis JA, Vodak P, Wilmore HH, et al.: Anaerobicthreshold and maximal aerobic power for three modes of exercise. J Appl Physiol, 1976, 41: 544-550. Medline

4) ACSM: ACSM's Guidelines for Exercise Testing and Prescription by American College of Sports Medicine $6^{\text {th }}$ edition. Philadelphia: Lippincott Williams \& Wilkins, 2006, pp 1-99.

5) McMaster WC, Stoddard T, Duncan W: Enhancement of blood lactate clearance following maximal swimming. Am J Sports Med, 1989, 17: 472-477. Medline] CrossRef

6) Lim EK, Kim MJ, Sin SY, et al.: Effect of recovery on dynamic strength after isotonic or isometric cool-down exercise. J Kor Acad Uni Train Phys Thera, 1994, 1: 67-74.

7) Wee SD: The response specificity of energy substrate and metabolic hormone during submaximal exercise. Sport Sci, 1995, 18: 201-209.

8) Lee WJ, Kim YH: The effect of massage after anaerobic exercise for blood lactate concentration. Exer Sci, 2002, 7: 249-257.

9) Bonen A, Belcastro AN: Comparison of self-selected recovery methods on lactic acid removal rates. Med Sci Sports, 1976, 8: 176-178. Medline [CrossRef

10) Ok JS: The effect of exercise recovery on metabolic and hormonal responses during the postexercise recovery period. KAHPERD, 1997, 19: 276-286.

11) Lee $\mathrm{TH}$, Lee $\mathrm{CH}$ : The effcets of sports massage on improving of low back pain. J Kor Well, 2008, 3: 31-36. 